\title{
Publisher's Note: Coupling between phonon-phonon and phonon-impurity scattering: A critical revisit of the spectral Matthiessen's rule [Phys. Rev. B 92, 235206 (2015)]
}

Tianli Feng, Bo Qiu, and Xiulin Ruan

(Received 30 March 2016; published 13 April 2016)

DOI: 10.1103/PhysRevB.93.159904

This paper was published online on 16 December 2015 with an incorrect reference. Reference [34] should read as "P. Carruthers, Phys. Rev. 126, 1448 (1962)." The reference has been corrected as of 31 March 2016. The reference is incorrect in the printed version of the journal. 